\title{
Numerical Simulation of the Effects of Design Parameters on the Performance of Tractor Powered Flail Choppers
}

\author{
Zhiqiang Zhang (0000-0002-1009-2178) ${ }^{1}$, Hongwen $\mathrm{Li}^{1}$, Allen D. McHugh², Jin He ${ }^{(\bowtie)}$, Qingjie Wang ${ }^{1}$, \\ Caiyun $\mathrm{Lu}^{1}$, Wenzheng $\mathrm{Liu}^{1}$, and Sun Nina ${ }^{1}$ \\ ${ }^{1}$ Beijing Key Laboratory of Optimized Design for Modern Agricultural Equipment, College of Engineering, China \\ Agricultural University, Beijing, China \\ \{zhangzhiqiang, lihongwen, hejin, wangqingjie, lucaiyun, lwzheng, \\ sunnina92\}@cau.edu.cn \\ ${ }^{2}$ International Maize and Wheat Improvement Center, Dhaka, Bangladesh \\ A.McHugh@cgiar.org
}

Abstract: Several design parameters of flail chopper were studied under a wide range of field and crop conditions to improve chopper performance. However, the effects of parameters on the internal straw flow characteristics that influence straw chopping and spreading performance are still unknown. In this paper, Computational Fluid Dynamics (CFD) were used to analyze the influence of three operating parameters (rotor speed, forward speed and hood clearance) and two structural parameters (fan blade number and flail tip clearance) on the flow characteristic in the tractor powered flail chopper. The pressure contours, maximum negative pressure and flow rate at the machine inlet were considered in the analysis of feeding performance. Velocity at 21 sampling points, area weighted and maximum velocities at the outlet were also considered for straw spreading performance. Streamlines from three sampling sections were analyzed for straw flow-ability.

Keywords: Flail chopper, Feeding performance, Spreading performance, Straw flow-ability, Design parameters

\section{Introduction}

Crop straw can be gathered in packs/bales for off-field utilization after harvesting, while for in-field utilization it is often mixed with soil or covered on the soil surface after being chopped and spread in the field by straw chopper. Crop straw field retention can reduce soil erosion, increase soil water content, and enhance crop productivity [1,2]. Furthermore, it can provide lots of environmental benefits such as elimination of residue burning and excessive use of fertilizers [3].

Residue retention practices and methods of dealing with excess plant residue commenced in China, in the 1980s. Nowadays as a consequence a number of flail choppers have been developed, and their performances have been studied though abundant experiments. Qiu et al. [4] studied the effects of rotor rotating speed and the space between rotary blade and stationary blade on straw length and power consumption through bench tests. Singh et al. [5] researched the optimal combination of straw moisture content, rotor rotating speed and forward speed on the basis of chopped straw size, straw spreading uniformity and fuel consumption through in-field experiment. Chen et al. [6] studied the influences of hood angle, feeding velocity and distances between blades 
on chopping quality and power requirement of the straw chopping devices. Shi et al. [7] designed a cotton straw chopper and analyzed the impact of rotor speed, forward speed and hood clearance on the length of chopped straw and the height of chopped rootstalk.

Fluid flow characteristics in the hood of the flail chopper have significant influence on its performance [8]. The fluid flow in the hood during operation is complex, which makes it difficult to acquire three-dimensional flow fields using traditional methods. Computer Fluid Dynamics (CFD) have been wildly applied in designing and optimizing agricultural equipment $[9,10,11]$.

Ayli et al. [12] aided by CFD, investigated the effects of blade angle, runner inlet and outlet diameters, and blade height on the performance and cavitation characteristics of turbine design. Zhang et al. [13] developed a CFD model to analyze the performance of the pressure regulator by considering the influence of inlet pressure and regulation assembly. Shen et al. [14] simulated the flow field during the pulverizing process within the crushing chamber, which revealed the influences of impeller speed, impeller blade shape and blade angle on the flow field. Wang et al. [15] constructed a three-dimensional model of chopped straw flow to simulate the flow fields and determine the influence of flail structural parameters. Chon et al. [16] investigated the effects of blade and hood design on flow patterns inside a counter rotating double-spindle lawn mower deck by experiment and computation.

These and many other researchers have studied the influence of flail chopper structural and operating parameters on straw chopping and spreading performance. However, very few studies have been conducted to determine the effect of design parameters on the fluid flow mechanisms in the flail chopper, and their effect on flail chopper performance. Utilizing CFD technology this paper developed a mathematical model to simulate the fluid flow characteristics under the hood of a tractor powered flail chopper. Based on steady state CFD analysis, the effects of three operating parameters (rotor speed $\omega$, forward speed $V$ and hood clearance $H$ ) and two structural parameters (fan blade number $N$, flail tip clearance $\varepsilon_{1}$ ) of the tractor powered flail chopper on fluid flow characteristics were obtained. The inlet flow rate, maximum negative pressure and pressure contours at inlet were chosen to analyze the straw feeding performance. The area-weight average velocity, maximum velocity and 21 sampling points at outlet were chosen to analyze spreading performance of the tractor powered flail chopper. Streamlines on three sampling sections in the hood were chosen to analyze the straw flow-ability.

\section{The Model of Three-Dimensional Global Fluid Field}

\subsection{Physical Model}

The tractor powered flail chopper (Fig. 1) is mainly composed of a hood, a chopping device (rotor, flails and fan blades), transmission, adjustable wheels and a support link. The transmission is used to transmit power to chopping device from power take off (PTO). The chopping device chops the feeding straw and disperses the chopped straw out of the hood. The machine is hitched to tractor by the support link. 


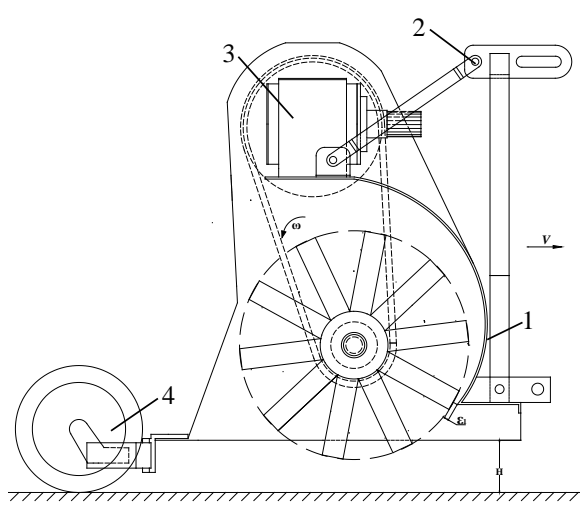

(a) Side view

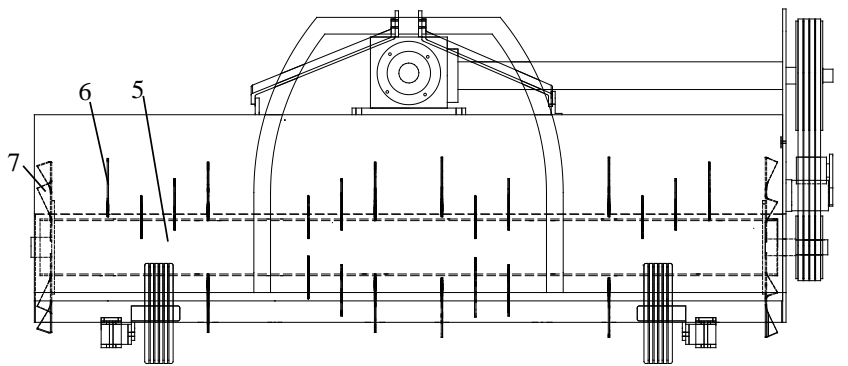

(b) Front view

1. Hood 2. Support link 3. Transmission 4. Adjustable height wheels 5. Rotor 6. Flail 7. Fan blade

Fig.1. Structural schematic diagram of tractor powered flail chopper

In operation, the flails cut and drag the straw into the hood, then chop, tear, and macerate the cut straw under the concave hood, and finally spread the chopped straw on the ground at the outlet of the hood $[17,18]$.

A number of simulations were performed to find out the effect of rotor speed, forward speed, hood clearance, fan blade number and flail tip clearance on the straw flow field. The main design parameters for simulation are presented in Table 1.

Table 1. Main design parameters of the tractor powered flail chopper

\begin{tabular}{cc}
\hline Technical parameters & Design values \\
\hline Machine dimensions $\mathrm{L} \times \mathrm{W} \times \mathrm{H} / \mathrm{mm}$ & $2429 \times 1309 \times 1073$ \\
Working width $/ \mathrm{mm}$ & 2000 \\
Rotor radius $/ \mathrm{mm}$ & 271 \\
Number of flails & 42 \\
Rotor speed $(\omega) / \mathrm{rpm}$ & $1900 ; 2100 ; 2300$ \\
Forward speed $(V) / \mathrm{m} . \mathrm{s}^{-1}$ & $0.5 ; 1.0 ; 1.5$ \\
Hood clearance $(H) / \mathrm{mm}$ & $110 ; 130 ; 150$ \\
Fan blade number $(N)$ & $0 ; 6 ; 12$ \\
Flail tip clearance $\left(\varepsilon_{1}\right) / \mathrm{mm}$ & $10 ; 20 ; 30$ \\
\hline
\end{tabular}

\subsection{Mathematic Model}

\subsubsection{Governing Equations}

To simulate fluid flow characteristics in the tractor powered flail chopper, we assumed; steady, incompressible, viscous, turbulent, and adiabatic flow conditions. The governing laws of conservation of mass and momentum were used in the simulation of fluid flow characteristics of the tractor powered flail chopper.

The equation for mass conservation for steady and incompressible flows, in cartesian tensor form, is written as follows [19]:

$$
\frac{\partial}{\partial x_{i}}\left(\rho u_{i}\right)=0
$$


Where $u_{i}$ is the velocity component in the $i$ direction; $\rho$ is the fluid density.

The equation for momentum conservation for steady flows, in cartesian tensor form, is written as follows [20]:

$$
\frac{\partial}{\partial x_{j}}\left(\rho u_{i} u_{j}\right)=-\frac{\partial p}{\partial x_{i}}+\frac{\partial \tau_{i j}}{\partial x_{j}}+F_{i}
$$

where $p$ is the static pressure; $\tau_{i j}$ is the stress tensore; $F_{i}$ is the body force.

The continuous rotation of the rotor and geometric changes in the circumferential direction will generate swirling and rotating flow. A three-dimensional rotating model was built to simulate flow field in the tractor powered flail chopper. The realizable k- $\varepsilon$ turbulence model which has a great enhancement on the flow including strong streamline curvature, vortices, and rotation is used for turbulent flow.

The transport equations for $\mathrm{k}$ and $\varepsilon$ in the realizable $\mathrm{k}-\varepsilon$ turbulence model after Rohdin et al. [21] are;

$$
\frac{\partial\left(\rho u_{j} \varepsilon\right)}{\partial x_{j}}=\left(\mu+\frac{\mu_{t}}{\sigma_{\varepsilon}}\right) \nabla^{2} \varepsilon+C_{1} S \rho \varepsilon-C_{2} \frac{\rho \varepsilon^{2}}{k+\sqrt{v \varepsilon}}
$$

where $C_{1}=\max [0.43, \eta /(\eta+5)], C 2=1.0, \sigma_{\varepsilon}=1.2$.

\subsubsection{Boundary Conditions}

To solve the motion equations for specific flow, initial and boundary conditions are normally applied. In this study, initial condition was not of concern as it was considered a steady-state problem. However, the boundary conditions which specified the values of dependent variables at the boundaries of the fluid domain were required to solve the flow in the chopper.

No-slip condition and standard wall functions were used at the walls and the flails. The pressure inlet boundary condition at the chopper inlet was set with a gauge pressure at $0 \mathrm{~Pa}$. The pressure outlet boundary condition was also set at $0 \mathrm{~Pa}$. Under rotation, an interface between the rotor and the hood was formed (Fig. 2).

\subsubsection{Computational Domain and Mesh Generation}

In order to simplify the computational model, some characteristics and components of the common tractor powered flail chopper were ignored during the building of the computational domain. The computational domain (Fig. 2) consisted of the hood and flail rotor, and the lower side of the hood was extended all the way to the ground [16]. In the calculations, the flail rotor was set in a rotary frame which was based on a multiple reference frame (MRF) model [22]. 


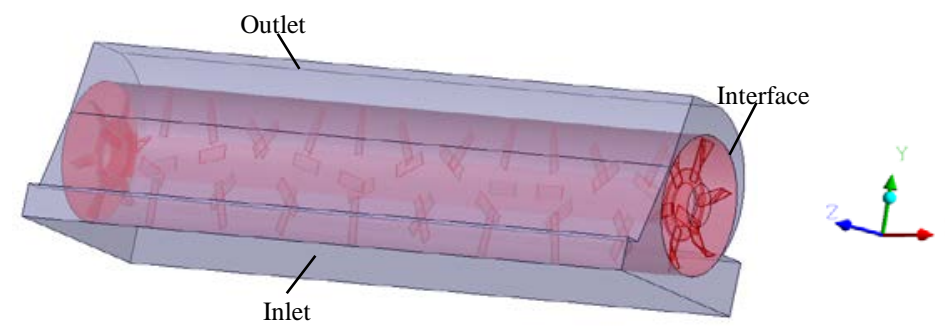

Fig.2. Computational domain of the tractor powered flail chopper

On account of the complexity of the tractor powered straw chopper geometry, tetrahedral mesh was generated to simulate internal flow field of the flail chopper model. Then, the tetrahedral mesh was transformed into polyhedral mesh (Fig. 3), which can reduce mesh number and improve mesh quality.

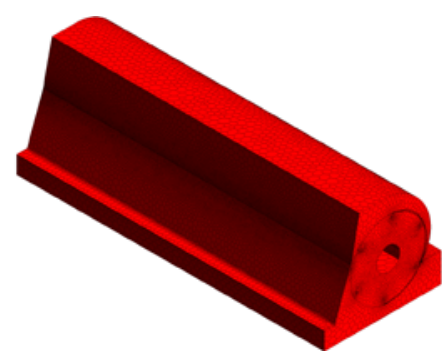

(a) Hood

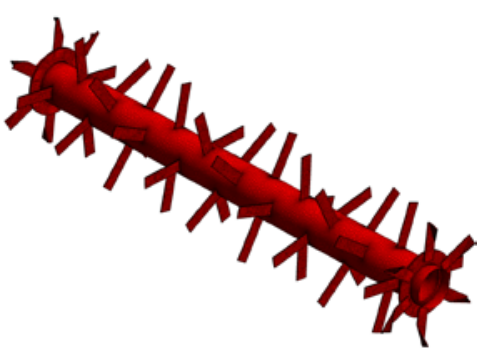

(b) Flail rotor

Fig.3. Polyhedral mesh of the flail chopper model

\subsection{Sampling Sections and Points}

The existence of flails and fan blades on the rotor cause differences in axial flow under the hood. Three sampling sections P1-P3 (Fig. 4a) and 21 other sampling points (Fig. 4b) were selected to enhance analysis of the fluid flow characteristic under the hood. Section P1 paralleled the Y-Z plane and passed through the left-side fan blades; section P2 was the symmetry plane; section P3 paralleled the Y-Z plane and passed through the right-side flails. Sampling Points 1-7 were located at the top of outlet plane; points 8-14 were located in the center of the outlet plane; points 15-21 were located at the base of the outlet plane.

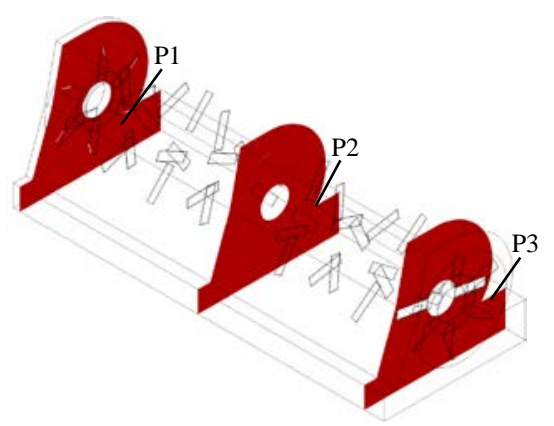

(a) Sampling sections

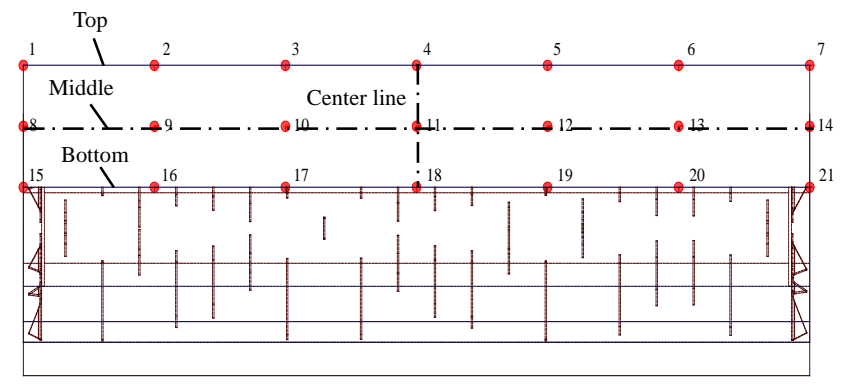

(b) Sampling points

Fig. 4. Location of the sampling sections and points

\section{Results and Discussions}

\subsection{The E $\square$ ect of Rotor Speed on Flail Chopper Performance}


The rotor speed is one of the most important operating parameters that affects the performance of the tractor powered flail chopper [23]. The rotor speed was set as 1900, 2100 and $2300 \mathrm{rpm}$ during the simulation, while all other design parameters were kept constant to test the e rotat ofeed on the tractor powered flail chopper performance.

\subsubsection{The E $\square$ ect of Rotor Speed on Straw Feeding Performance}

The negative pressure field, maximum negative pressure and flow rate at inlet under different rotor speeds was shown in Fig. 5 and Fig. 6. Large areas of negative pressure were found at the inlet under different rotor speeds. The pressure on the left and right sides of the hood was lower than that of the surrounding areas because of the existence of fan blades on the left and right sides of the rotor. The maximum negative pressure was $14.80 \mathrm{~Pa}$ when the rotor was set at $2300 \mathrm{rpm}$, while the increased rotor speed increased the maximum negative pressure and negative pressure area at chopper inlet. The inlet flow rate increased with increased rotor speed, and the maximum inlet flow rate was $0.3877 \mathrm{~kg} \cdot \mathrm{s}^{-1}$ when the rotor was set at $2300 \mathrm{rpm}$. With increased negative pressure and inlet flow rate, more crop straw was drawn into the chopper for processing. Thus, increased rotor speed improved straw feeding performance at the inlet of the chopper.

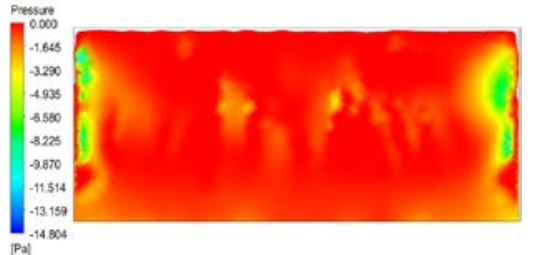

(a) $\omega=1900 \mathrm{rpm}$

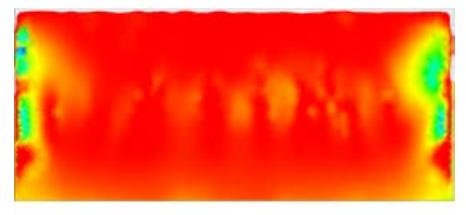

(b) $\omega=2100 \mathrm{rpm}$

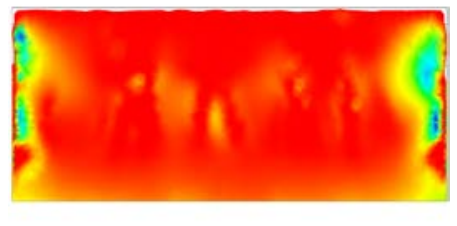

(c) $\omega=2300 \mathrm{rpm}$

Fig. 5. Pressure contours (Pressure $\leq 0 \mathrm{~Pa}$ ) at inlet of the chopper under different rotor speeds

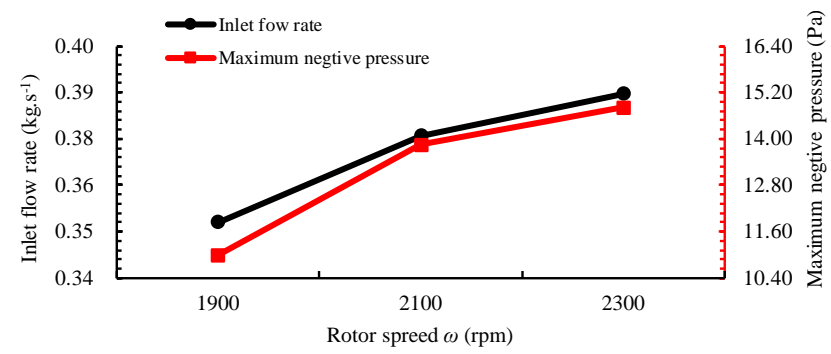

Fig. 6. Flow rate and maximum negative pressure at the inlet under different rotor speeds

\subsubsection{The E $\square$ ect of Rotor Speed on Straw Spreading Performance}

Fig. 7 showed the area-weighted average and maximum velocities at outlet under different rotor speeds. As rotor speed increased both the area-weighted average velocity and maximum velocity increased. The area-weighted average velocity increased by $7.59 \%$ and $11.10 \%$ as the rotor speed increased from $1900 \mathrm{rpm}$ to $2100 \mathrm{rpm}$ and from $2100 \mathrm{rpm}$ to $2300 \mathrm{rpm}$, respectively. Maximum velocity increased by $10.18 \%$ and $7.61 \%$ when the rotor speed was changed from 1900 rpm to $2100 \mathrm{rpm}$ and from $2100 \mathrm{rpm}$ to $2300 \mathrm{rpm}$, respectively. 


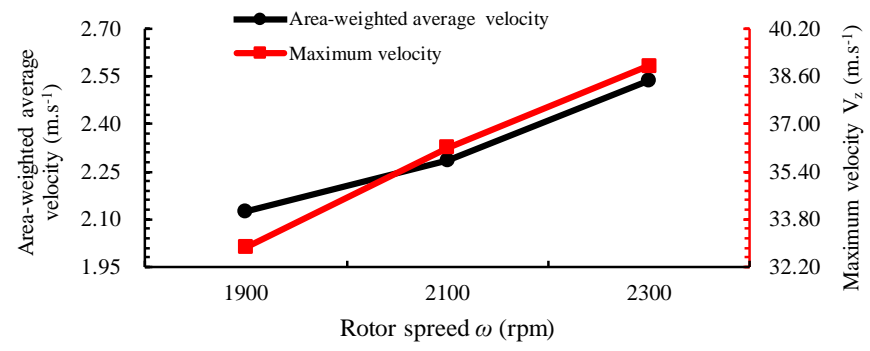

Fig. 7. Area-weighted average and maximum velocities at outlet under different rotor speeds

The velocity at all 21 sampling points at the outlet pane increased as the rotor speed increased from $1900 \mathrm{rpm}$ to $2300 \mathrm{rpm}$ (Fig. 8). The velocity increased from top to bottom of the outlet plane under same rotor speed, and the velocity was generally symmetric from the center line at the outlet plane. The velocity distribution curve was similar under different rotor speeds at the top, middle and bottom of the outlet plane. The velocity at the top of the outlet plane except at point 4 was similar at same rotor speed, and the velocity at center area was higher than that of all other points. The velocity at the center of the outlet plane, except point 8 and point 14 was similar at same rotor speed, and the velocity on the right and left side areas were higher than that at other places because of the fan blades. However, there was an obvious velocity variation at the bottom of the outlet plane under the same rotor speed.

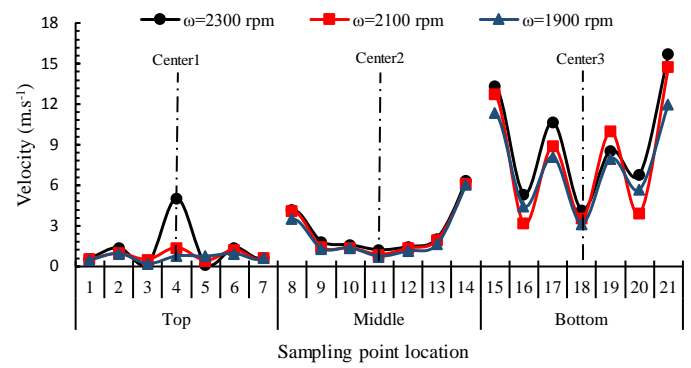

Fig. 8. Velocity at sampling points under different rotor speeds

According to our observations, increased rotor spread increased the area-weighted average velocity, maximum and sampling points' velocity at outlet plane, but rotor speed had limited influence on velocity distribution. Thus, increased rotor speed can improve straw spreading performance. Our observations agree with the findings of Ma et al. [24] where it was shown that the rotor speed had a significant influence on the straw spreading distance, and the straw spreading distance could be increased by increasing the rotor speed.

\subsubsection{The E $\square$ ect of Rotor Speed on Straw Flow-ability}

The streamlines at P1, P2 and P3 under various rotor speeds were shown in Fig. 9. As indicated in Fig.9a vortices were distributed to the front section of the inlet region and the lower back section of the hood. Rotor speed had negligible influence on the size and position of the vortex regions and streamline distribution at outlet plane. The results showed that increased rotor speed had limited influence on the straw flow-ability at right and left side of the hood.

Vortices were distributed to the lower front and back of the hood at 1900 rpm rotor speed, and a recirculation zone appeared at outlet (Fig. 9b). When rotor speed was increased from 1900 rpm to $2100 \mathrm{rpm}$, the vortex at the lower back of the hood and recirculation zone at outlet 
diminished. The vortex at the lower front of the hood disappeared when the rotor speed increased from $2100 \mathrm{rpm}$ to $2300 \mathrm{rpm}$, but a vortex region at outlet appeared. The results indicated that increased rotor speed might improve straw flow-ability in the middle area of the hood.

Fig.9c indicated that the vortices were distributed at the lower front and back of the hood. The size of the vortex region at the lower front of the hood decreased as rotor speed increased, but rotor speed had little influence on the size and position of the vortex region at the lower back of the hood. The results indicated that increased rotor speed might improve straw flow-ability around flail blade areas.
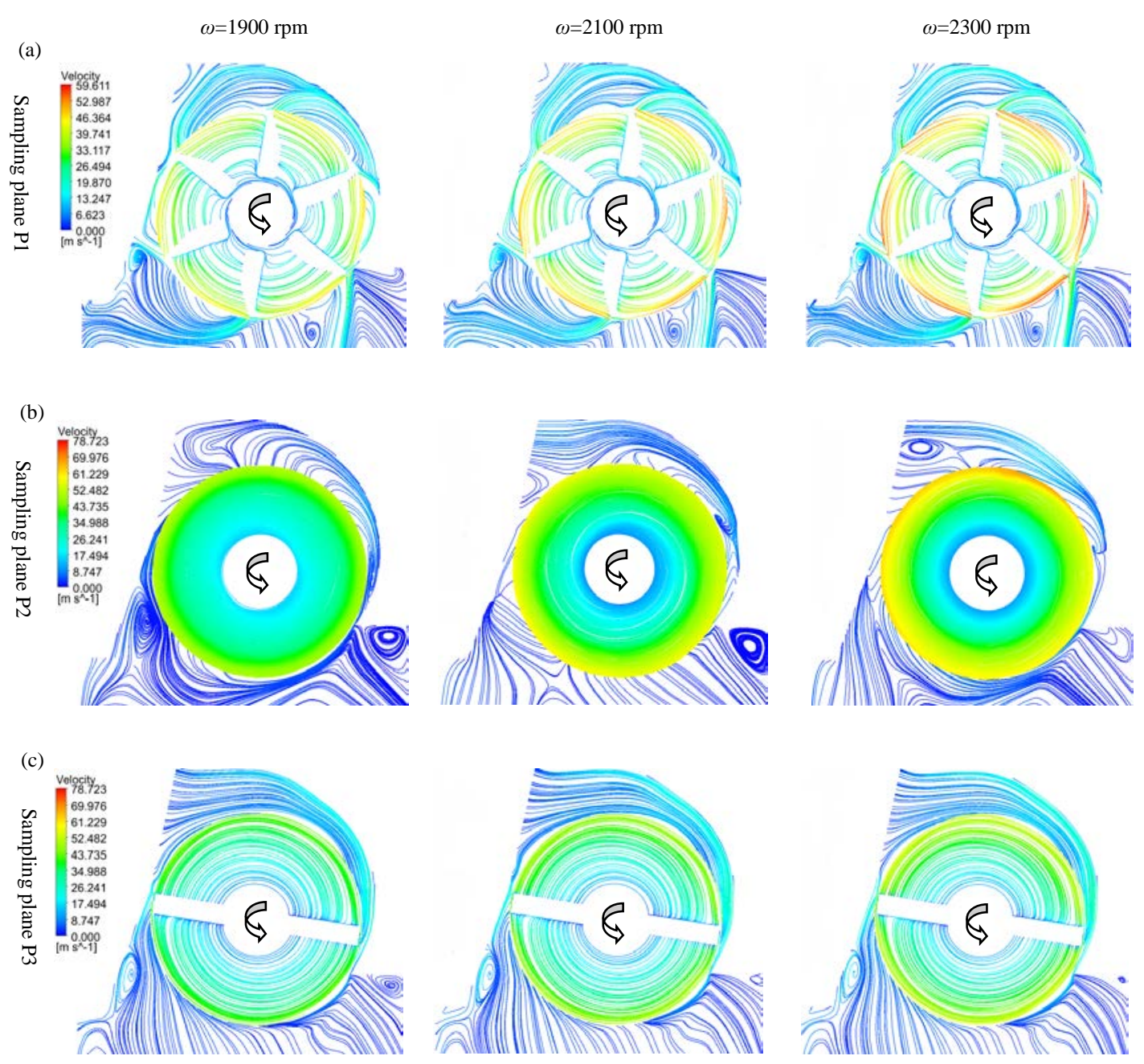

Fig. 9. Streamlines at P1, P2 and P3 at different rotor speeds

According to the analysis of the three sampling sections, it can be concluded that the increase of rotor speed might improve straw flow-ability in the hood.

\subsection{The E $\square$ ect of Forward Speed on Flail Chopper Performance}

The forward speed is one of the operating parameters that is important to the work efficiency and work quality of the tractor powered flail chopper [5]. The forward speed $V$ was set as $0.5,1.0$ and $1.5 \mathrm{~m} . \mathrm{s}^{-1}$, while all other design parameters were kept constant to test the e foeutaod speed on tractor powered flail chopper performance.

\subsubsection{The E $\square$ ect of Forward Speed on Straw Feeding Performance}


The negative pressure field, maximum negative pressure and flow rate at inlet under different forward speeds were shown in Fig. 10 and Fig. 11. Large areas of negative pressure were produced at the inlet under different forward speeds, especially on the left and right sides of the hood. The maximum negative pressure was $18.72 \mathrm{~Pa}$ for a forward speed of $0.5 \mathrm{~m} . \mathrm{s}^{-1}$, and an increase in forward speed decreased the maximum negative pressure at inlet of the hood. An increase of forward speed decreased the inlet flow rate, and the maximum inlet flow rate was $0.3875 \mathrm{~kg} . \mathrm{s}^{-1}$. Therefore, increased forward speed would degrade straw feeding performance at the inlet of the hood.

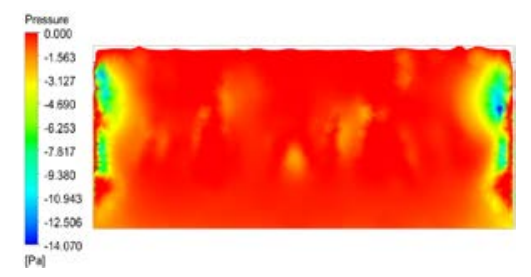

(a) $V=0.5 \mathrm{~m} . \mathrm{s}^{-1}$

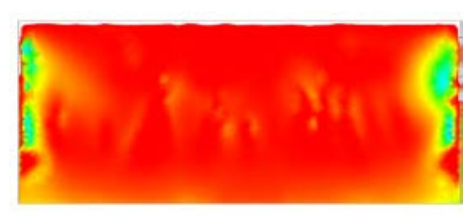

(b) $V=1.0 \mathrm{~m} \cdot \mathrm{s}^{-1}$

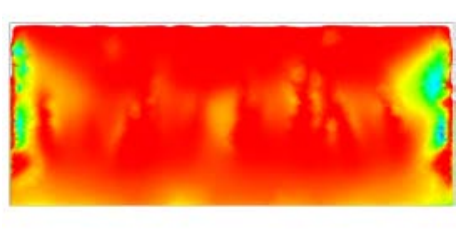

(c) $V=1.5 \mathrm{~m} \cdot \mathrm{s}^{-1}$

Fig. 10. Pressure contours (Pressure $\leq 0 \mathrm{~Pa}$ ) at inlet of the chopper under different forward speeds

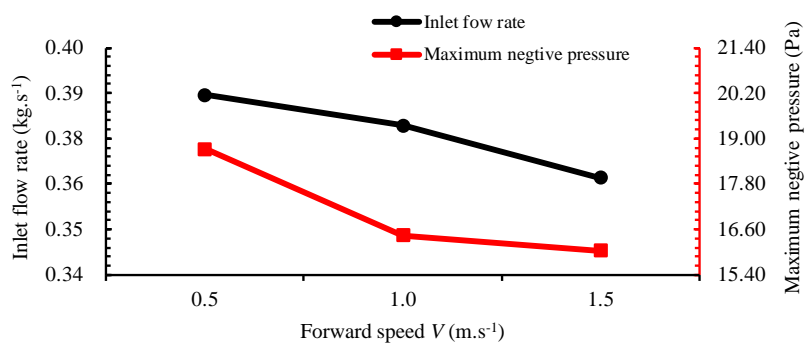

Fig. 11. Flow rate and maximum negative pressure at the inlet under different forward speeds

\subsubsection{The E $\square$ ect of Forward Speed on Straw Spreading Performance}

Fig. 13 indicated the area-weighted average and maximum velocities at the outlet under different forward speeds. The area-weighted average velocity decreased by $13.25 \%$ and $8.74 \%$ as the forward speed changed from $0.5 \mathrm{~m} . \mathrm{s}^{-1}$ to $1.0 \mathrm{~m} . \mathrm{s}^{-1}$ and from $1.0 \mathrm{~m} . \mathrm{s}^{-1}$ to $1.5 \mathrm{~m} . \mathrm{s}^{-1}$, respectively. The maximum velocity decreased (4.49\%) when the forward speed increased from $0.5 \mathrm{~m} . \mathrm{s}^{-1}$ to 1.0 $\mathrm{m} . \mathrm{s}^{-1}$ but increased marginally $(0.16 \%)$ when forward speed increased from $1.0 \mathrm{~m} . \mathrm{s}^{-1}$ to $1.5 \mathrm{~m} . \mathrm{s}^{-1}$.

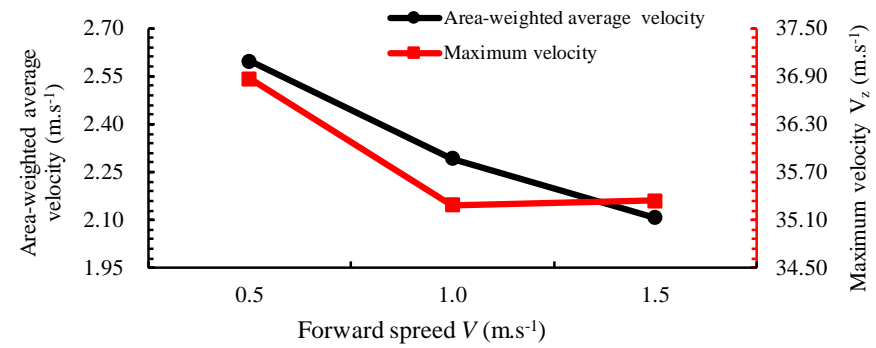

Fig. 13. Area-weighted average and maximum velocities at the outlet under different forward speeds

The velocity at 21 sampling points under different forward speeds as shown in Fig. 14 indicated velocity increased from top to bottom of the outlet plane at same forward speed, and the velocity was generally symmetric of center line at the outlet plane. The velocity distribution curve was similar under different forward speeds at the top, middle and bottom of the outlet plane. The 
velocity at the top of the outlet plane, except at point 4 , was similar at the same forward speed, and the velocity at center area was higher than that of other sampling points. The velocity at the middle of the outlet plane except at point 8 and point 14 was similar at the same forward speed, and the velocity at right and left side areas was higher than that at other sampling points. However, there was an obvious velocity variation at bottom of the outlet plane at same forward speed.

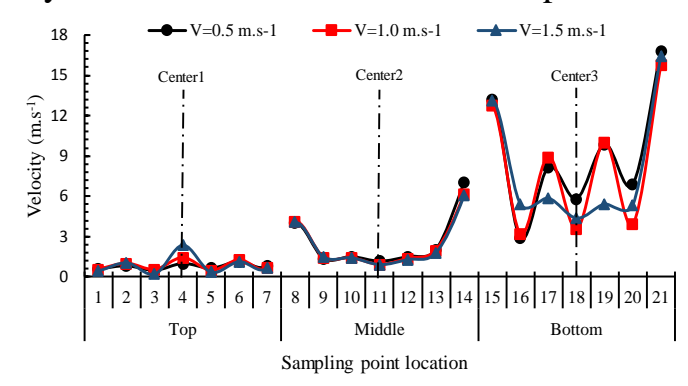

Fig. 14. Velocity at sampling points under different forward speeds

According to the above observations, it can be concluded that forward speed might have little influence on straw spreading performance. These results are in agreement with those obtained by Singh et al. [5] who found that there was no significant effect of forward speed on straw spreading uniformity at $5 \%$ level of significance.

\subsubsection{The E $\square$ ect of Forward Speed on Straw Flow-ability}

The streamlines on P1, P2 and P3 under different forward speeds were shown in Fig. 15. Fig.15a showed that the vortices were distributed at the front part of the inlet region and the lower back of the hood, and the size of the vortex region at the front part of the inlet region decreased with an increase of the forward speed. The results showed that the increase of the forward speed might improve straw flow-ability at right and left side of the hood.

Fig.15b showed that the vortices were distributed at the lower back of the hood and the outlet area when the forward speed was set at $0.5 \mathrm{~m} . \mathrm{s}^{-1}$. When the forward speed was increased from 0.5 $\mathrm{m} .{ }^{-1}$ to $1.0 \mathrm{~m} \cdot \mathrm{s}^{-1}$, the vortex at the outlet area and the back of the hood disappeared, but a vortex appeared at the lower front of the hood. A vortex reemerged when the forward speed was increased from $1.0 \mathrm{~m}^{-1}$ to $1.5 \mathrm{~m} \cdot \mathrm{s}^{-1}$. The results showed that straw flow-ability at the middle area of the hood would be improved with an increase in forward speed from $0.5 \mathrm{~m}^{-1}$ to $1.0 \mathrm{~m} . \mathrm{s}^{-1}$, but decreased as it was increased from $1.0 \mathrm{~m} \cdot{ }^{-1}$ to $1.5 \mathrm{~m} \cdot \mathrm{s}^{-1}$.

Fig.15c showed that the vortices were distributed at the lower back of the hood under different forward speeds. A vortex appeared at the lower front of the hood when the forward speed changed from $0.5 \mathrm{~m}^{-1}$ to $1.0 \mathrm{~m} . \mathrm{s}^{-1}$, the size of the vortex region expanded when the forward speed was increased from $1.0 \mathrm{~m} .{ }^{-1}$ to $1.5 \mathrm{~m} . \mathrm{s}^{-1}$. The results showed that an increase in forward speed might decrease straw flow-ability around flail areas. 

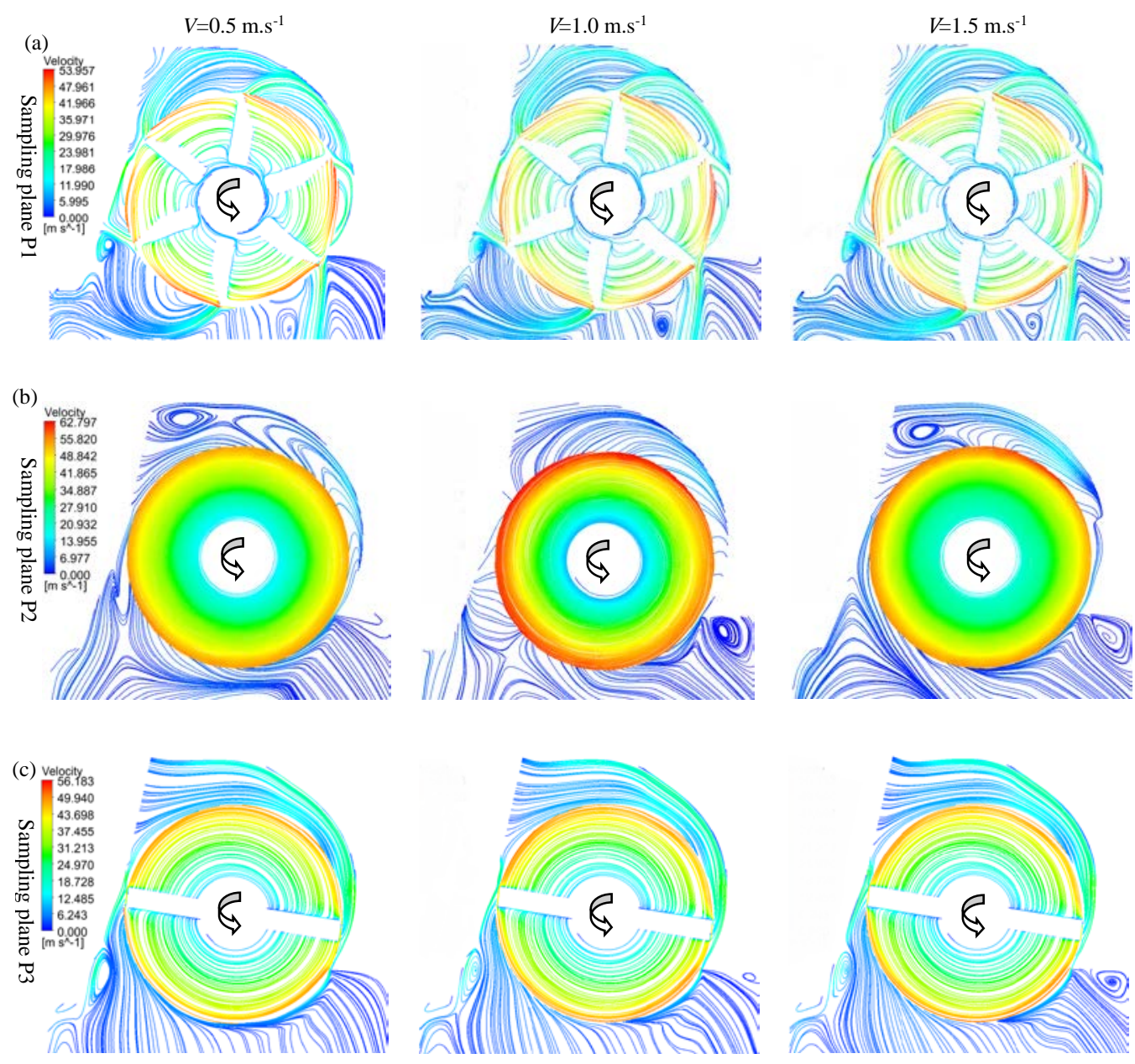

Fig. 15. Streamlines on $\mathrm{P} 1, \mathrm{P} 2$ and $\mathrm{P} 3$ under different forward speeds

According to the analysis of the three sampling sections, it can be concluded that the increase of forward speed might decrease straw flow-ability in the hood.

\subsection{The E $\square$ ect of Hood Clearance on Flail Chopper Performance}

The hood clearance is an operating parameter that is important to stubble height and straw flow rate [25]. The hood clearance was set as 110, 130 and $150 \mathrm{~mm}$, while all other design parameters were kept constant to test the e howd ofearance on tractor powered flail chopper performance.

\subsubsection{The E $\square$ ect of Hood Clearance on Straw Feeding Performance}

The negative pressure field, maximum negative pressure and flow rate at inlet under different hood clearances was shown in Fig. 16 and Fig. 17. Large areas of negative pressure were produced at the inlet under different hood clearances, and the negative pressure at left and right sides of the chopper was larger than surrounding areas. The maximum negative pressure was $35.53 \mathrm{~Pa}$ when the hood clearance was set at $110 \mathrm{~mm}$, and with an increase in hood clearance it decreased the maximum negative pressure at inlet of the hood. The inlet flow rate firstly increased and then decreased as the hood clearance increased, and the maximum inlet flow rate was $0.3768 \mathrm{~kg} . \mathrm{s}^{-1}$. It was concluded that too large or too small hood clearance might degrade straw feeding performance at the inlet of the chopper. 


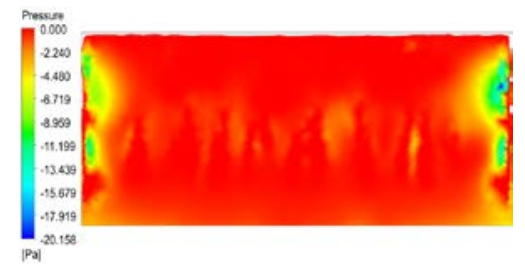

(a) $H=110 \mathrm{~mm}$

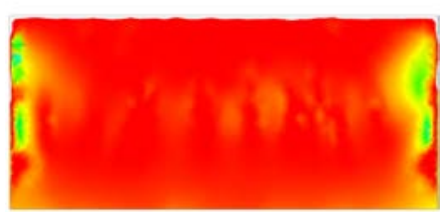

(b) $H=130 \mathrm{~mm}$

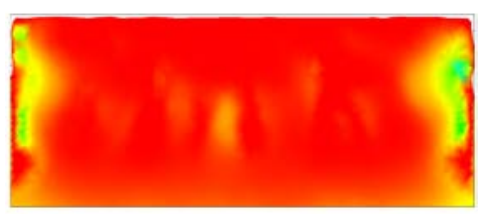

(c) $H=150 \mathrm{~mm}$

Fig. 16. Pressure contours (Pressure $\leq 0 \mathrm{~Pa}$ ) at inlet of the chopper with different hood clearances

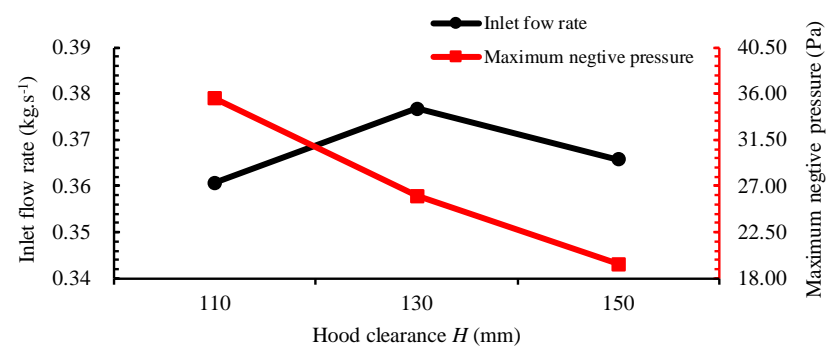

Fig. 17. Flow rate and maximum negative pressure at inlet under different hood clearances

\subsubsection{The E $\square$ ect of Hood Clearance on Straw Spreading Performance}

Fig. 18 indicated the area-weighted average and maximum velocity at the outlet under different hood clearances. With an increase in hood clearance, the area-weighted average velocity increased. The area-weighted average velocity increased by $3.11 \%$ and $29.83 \%$ as the hood clearance was changed from $110 \mathrm{~mm}$ to $130 \mathrm{~mm}$ and from $130 \mathrm{~mm}$ to $150 \mathrm{~mm}$, respectively. Maximum velocity increased by $1.11 \%$ when the hood clearance increased from $110 \mathrm{~mm}$ to 130 mm but decreased by $2.74 \%$ when the hood clearance changed from $130 \mathrm{~mm}$ to $150 \mathrm{~mm}$.

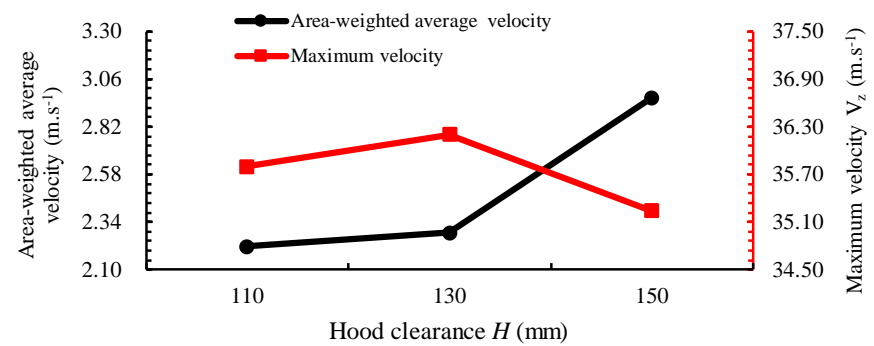

Fig. 18. Area-weighted average and maximum velocities at outlet under different hood clearances

The velocity at 21 sampling points under different hood clearances was shown in Fig. 19. The velocity increased from top to bottom of the outlet plane, and the velocity was generally symmetric of center line at the outlet plane under same hood clearance. The velocity distribution curve was similar under different hood clearances at the top, middle and bottom of the outlet plane. The velocity with a clearance of $150 \mathrm{~mm}$ was usually higher than that with the hood clearance of 110 and $130 \mathrm{~mm}$. The velocity at the top of the outlet plane was similar at clearance of 130 and $150 \mathrm{~mm}$, but the velocity at point 4 was much larger than that at the other sampling points when hood clearance was $110 \mathrm{~mm}$. The velocity at middle of the outlet plane except at points 8 and 14 was similar at the same hood clearance, and the velocity in the right and left side areas was higher than that of other points. However, there was an obvious velocity variation at bottom of the outlet plane at same hood clearance. 


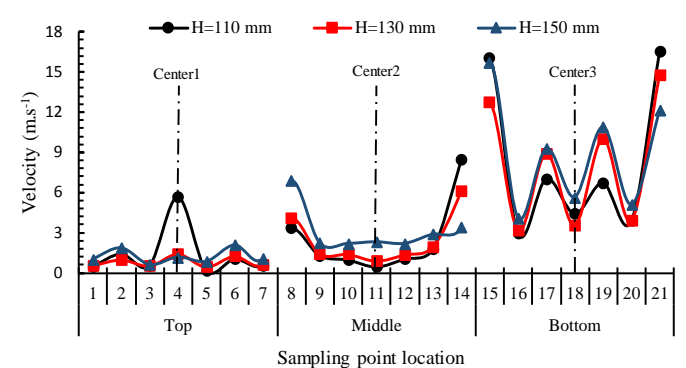

Fig. 19. Velocity at sampling points under different hood clearances

According to above analysis, it can be concluded that the increase of hood clearance might improve straw spreading performance.

\subsubsection{The E $\square$ ect of Hood Clearance on Straw Flow-ability}

The streamlines on P1, P2 and P3 under different hood clearances were shown in Fig. 20. Fig.20a outlined the vortices at the front part of the inlet region and the lower back of the hood, and this hood clearance had little influence on the size and position of the vortex regions. The results also showed that the hood clearance had little influence on the straw spreading performance at right and left side of the hood.

Fig.20b showed the appearance of a recirculation zone at outlet when hood clearance was 110 $\mathrm{mm}$. However, the recirculation zone dissipated as hood clearance increased from $110 \mathrm{~mm}$ to 130 $\mathrm{mm}$, with a vortex at the lower front of the hood appearing when the hood clearance increased from $130 \mathrm{~mm}$ to $150 \mathrm{~mm}$, however a vortex and a recirculation zone appeared at outlet. The results showed that an increase in hood clearance might improve straw flow-ability in the middle area of the hood.

Vortices were distributed at the lower back of the hood under different hood clearances (Fig. 20c). The vortex region at the lower back of the hood became smaller as the hood clearance changed from $110 \mathrm{~mm}$ to $130 \mathrm{~mm}$, but a small vortex region appeared at the lower front of the hood. The small vortex at the lower front of the hood disappeared when the hood clearance increased from $130 \mathrm{~mm}$ to $150 \mathrm{~mm}$, and the vortex region at the lower back of the hood became even smaller. The results showed that an increase of the hood clearance might improve straw flow-ability around the flail areas.
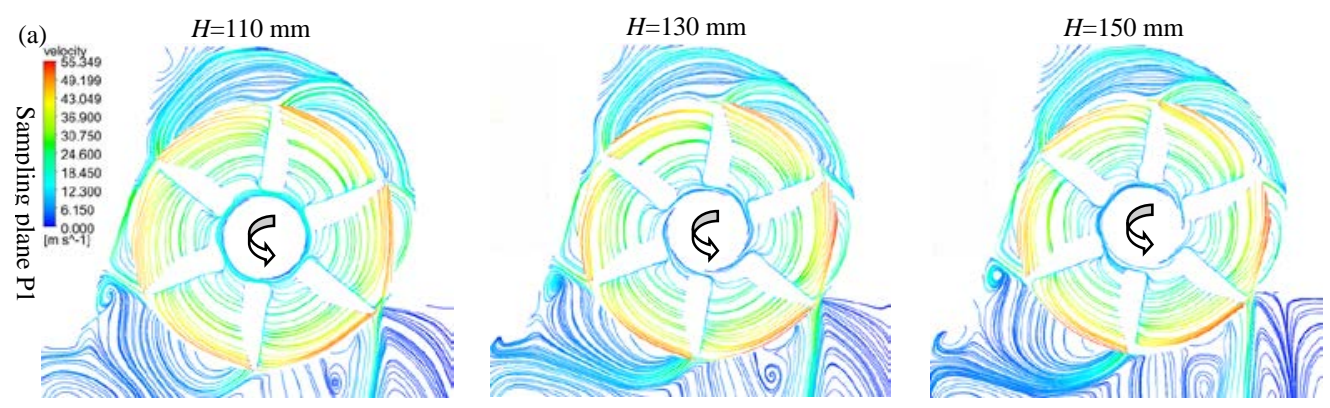

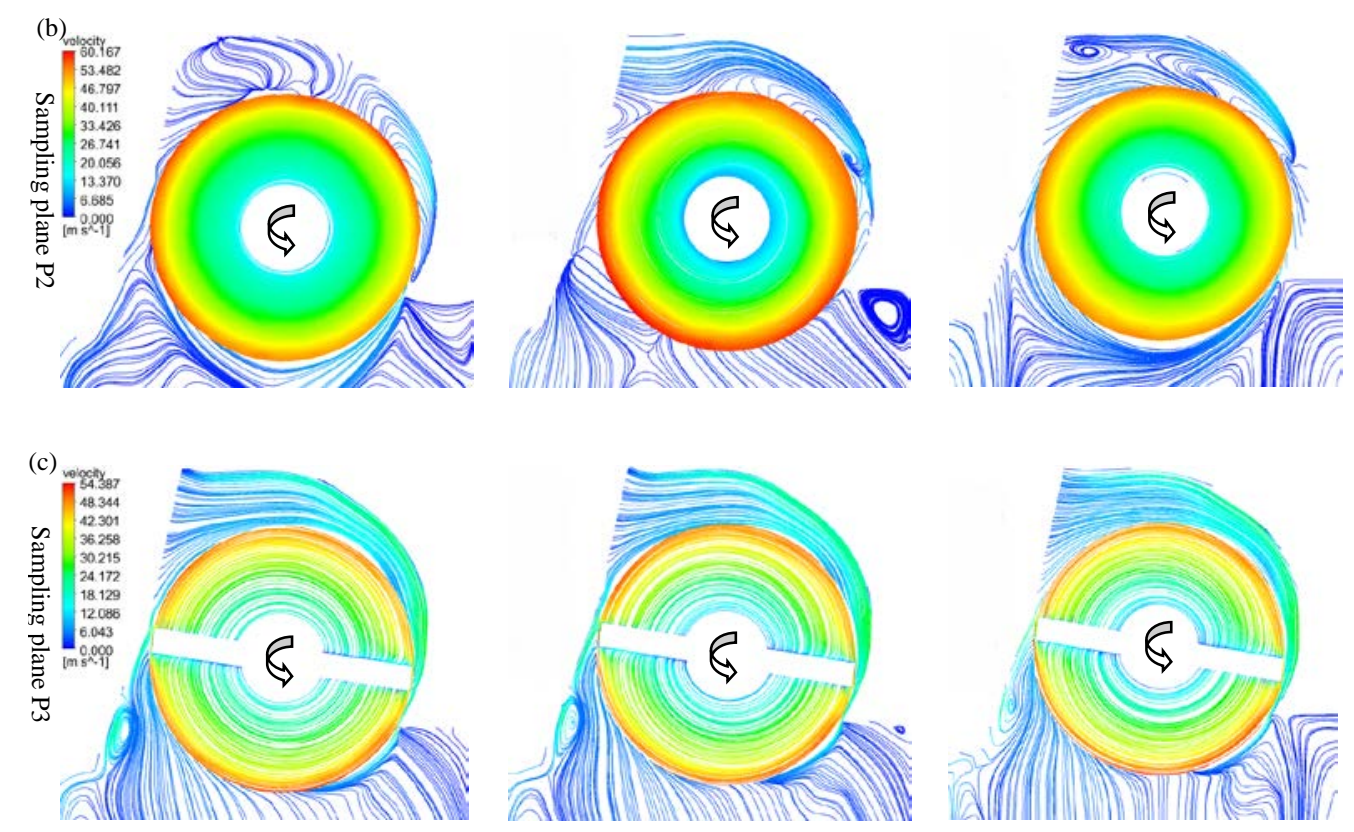

Fig. 20. Streamlines on $\mathrm{P} 1, \mathrm{P} 2$ and $\mathrm{P} 3$ under different hood clearances

\subsection{The E $\square$ ect of Fan Blade Number on Flail Chopper Performance}

The number of fan blades have an important effect on the performance of the flail choppers [26]. The number of fan blades was set as 0, 6 and 12, while all other design parameters were kept constant to test the e bledeoumber on tractor powered flail chopper performance.

\subsubsection{The E $\square$ ect of Fan Blade Number on Straw Feeding Performance}

The negative pressure field, maximum negative pressure and flow rate at inlet utilizing different blade numbers was shown in Fig. 21 and Fig. 22. Negative pressure zones were produced at the inlet under different blade numbers, and the existence of the fan blades increased the pressure at left and right sides of the hood. The maximum negative pressure was $26.02 \mathrm{~Pa}$ with 12 fan blades, and the increase in blade number significantly increased the maximum negative pressure and negative pressure area at inlet of the hood. Increased fan blade number significantly increased the inlet flow rate, and the maximum inlet flow rate was $0.3768 \mathrm{~kg} . \mathrm{s}^{-1}$. Thus, an increase in fan blade number can significantly improve the straw feeding performance at the inlet of the flail chopper.

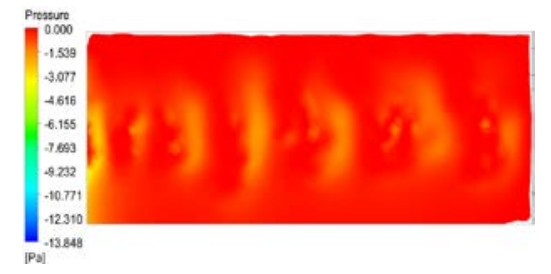

(a) $N=0$

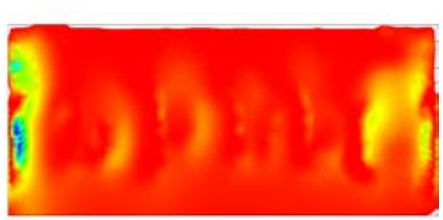

(b) $N=6$

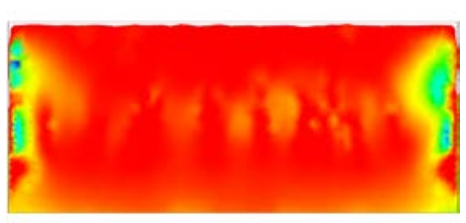

(c) $N=12$

Fig. 21. Pressure contours (Pressure $\leq 0 \mathrm{~Pa}$ ) at inlet of the chopper with different fan blade numbers 


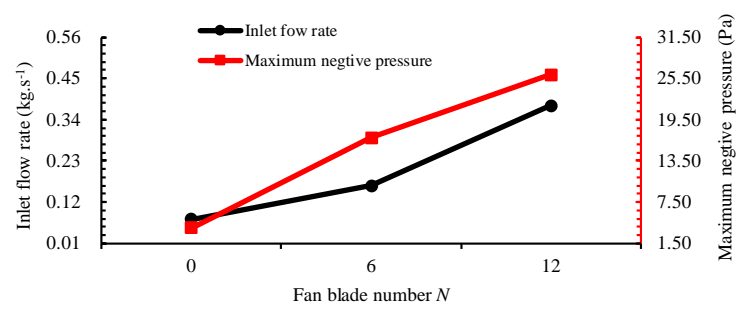

Fig. 22. Flow rate and maximum negative pressure at inlet with different fan blade numbers

\subsubsection{The E $\square$ ect of Fan Blade Number on Straw Spreading Performance}

An increase in fan blade number, improved area-weighted average and maximum velocities (Fig. 23). The area-weighted average velocity increased by $31.15 \%$ and $7.87 \%$ as blade number changed from 0 to 6 and from 6 to 12, respectively. The maximum velocity increased by 178.54\% and 11.12\% when blade number was increased from 0 to 6 and from 6 to 12, respectively. The existence of the fan-shaped blades significantly increased the maximum velocity and the area-weighted average velocity. However, increasing fan blade number from 6 to 12 did not significantly increase the maximum velocity and the area-weighted average velocity.

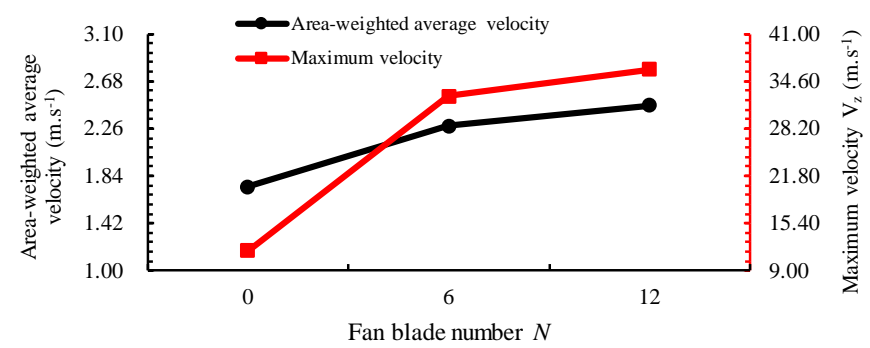

Fig. 23. Area-weighted average and maximum velocities at outlet with different fan blade numbers

The velocity at 21 sampling points under different fan blade numbers was shown in Fig. 24. The velocity increased from top to bottom of the outlet plane at the same fan blade number, and the velocity was generally symmetric of center line at the outlet plane. The velocity distribution curve was similar under different fan blade numbers at the top of the outlet plane. The velocity distribution curve was similar when the blade number was 6 and 12 at the middle and bottom of the outlet plane. The velocity at the top and middle of the outlet plane except at sampling points 1 , 7, 8 and 14 was similar when the fan blade number was set at 0 , and the velocity at right and left side areas was lower than that at other sampling points. The velocity at the top of the outlet plane except at point 4 was similar when blade number was set at 6 and 12, and the velocity at center areas was higher than other sampling points. The velocity at middle of the outlet plane, except points 8 and 14 was similar with 6 and 12 blades, and the velocity on the right and left side areas was higher than that at other sampling points. However, there was an obvious velocity variation at bottom of the outlet plane at the same fan blade number. It can be concluded that an increase in fan blade number would affect the velocity distribution uniformity at outlet plane. 


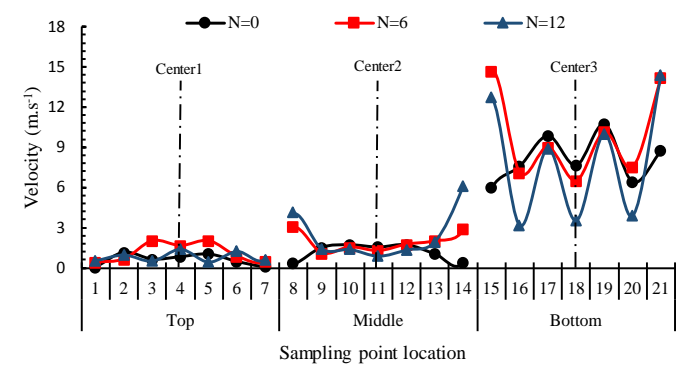

Fig. 24. Velocity at different sampling points with different fan blade numbers

According to these observations, increasing fan blade number would improve straw spreading performance.

\subsubsection{The E $\square$ ect of Fan Blade Number on Straw Flow-ability}

The streamlines on P1, P2 and P3 under different fan blade numbers were shown in Fig. 25. Fig.25a indicated that an increase in blade number decreased the fluid velocity around the rotor at P1. Additionally, a vortex was distributed at the lower back of the hood. The vortex dissipated when blade number was changed from 0 to 6 , but the number of streamlines passing through outlet plane decreased. When blade number was increased from 6 to 12, vortices appeared at the front part of the inlet region and the lower back of the hood. The results showed that an increase in fan blade number would degrade the straw spreading performance on right and left extremities of the hood.

Fig.25b showed the increase of the fan blade number and fluid velocity around the rotor at P2. Vortices were distributed at lower back and outlet of the hood when blade number was set at 0 . The vortex at lower back of the hood dissipated as blade number was changed from 0 to 6 , but two other vortices appeared near the rotor. When blade numbers were increased from 6 to 12, only one vortex was observed at lower front of the hood. The results showed that straw flow-ability in the middle area of the hood would be reduced as the fan blade number increased from 0 to 6 , but improved when blade number was increased from 6 to 12 .

Fig.25c was indicative of the increase in fan blade number increased the number of streamlines passing through the outlet plane and improved the streamline distribution uniformity at outlet. Vortices appeared at the lower front and back of the hood when blade number was increased from 6 to 12 .
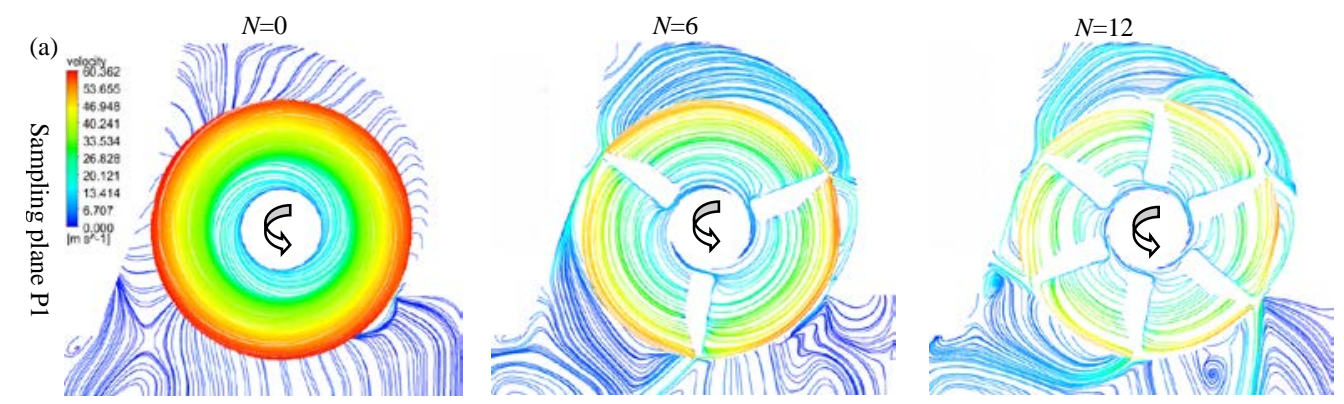

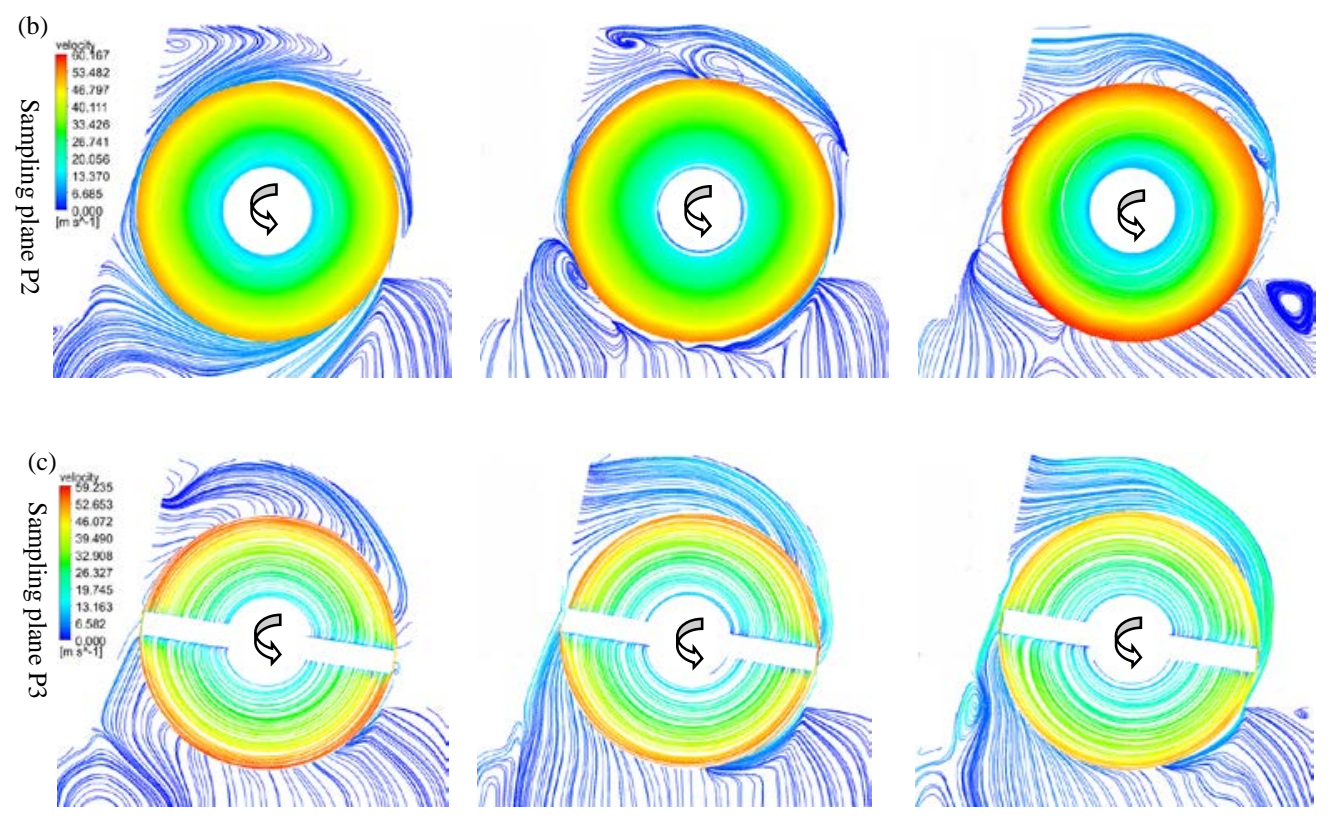

Fig. 25. Streamlines on P1, P2 and P3 under different fan blade numbers

According to the analysis of the three sampling sections, it can be concluded that an increase in fan blade number might reduce straw flow-ability in the shell.

\subsection{The E $\square$ ect of Flail Tip Clearance on Flail Chopper Performance}

Flail tip clearance is an important structural parameter for defining the hood geometry [27]. Flail tip clearance was set as 10, 20 and $30 \mathrm{~mm}$, while all other design parameters were kept constant to test the e

flæidttipf clearance on tractor powered flail chopper performance.

\subsubsection{The E $\square$ ect of Flail Tip Clearance on Straw Feeding Performance}

The negative pressure field, maximum negative pressure and flow rate at inlet under different flail tip clearances was shown in Fig. 26 and Fig. 27. Large areas of negative pressure were produced at the inlet under different tip clearances. The maximum negative pressure was $19.91 \mathrm{~Pa}$ when tip clearance was set at $30 \mathrm{~mm}$, and an increase in tip clearance increased the maximum negative pressure and negative pressure area at inlet of the hood. The inlet flow rate increased as tip clearance increased, and the maximum inlet flow rate was $0.6199 \mathrm{~kg} . \mathrm{s}^{-1}$. Thus, an increase in flail tip clearance can improve the straw feeding performance at the inlet.

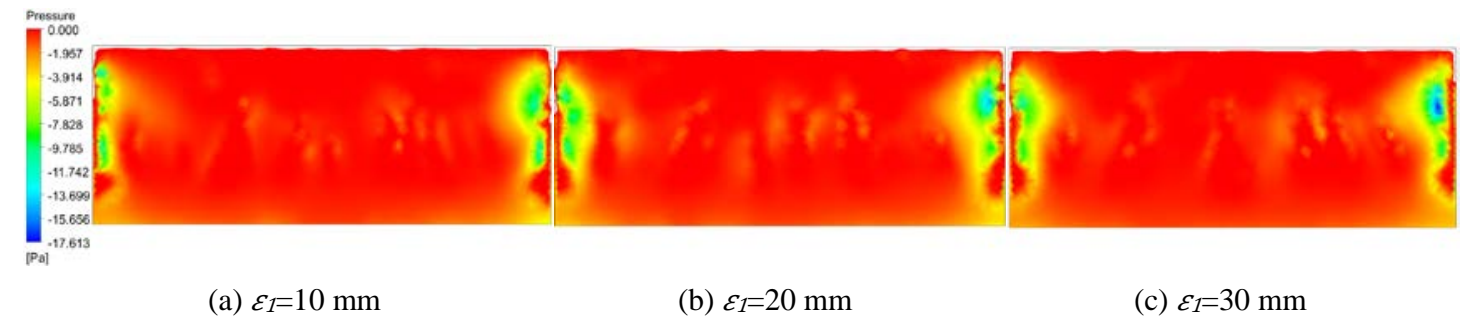

Fig. 26. Pressure contours (Pressure $\leq 0 \mathrm{~Pa}$ ) at inlet of the chopper under different flail tip clearances 


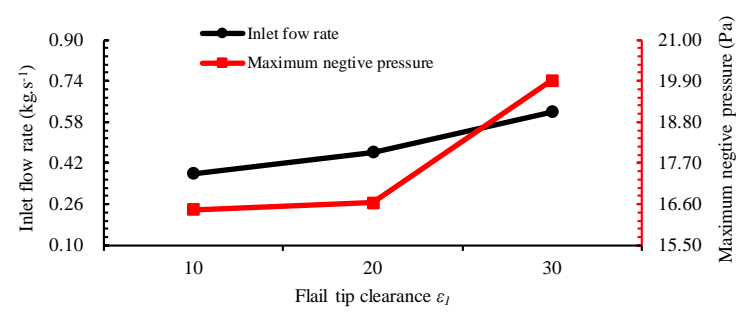

Fig. 27. Flow rate and maximum negative pressure at inlet under different flail tip clearances

\subsubsection{The E $\square$ ect of Flail Tip Clearance on Straw Spreading Performance}

Fig. 28 indicated the area-weighted average and maximum velocities at the outlet under different flail tip clearances. The area-weighted average and maximum velocities decreased with the increase in tip clearance. The area-weighted average velocity decreased by $4.78 \%$ and $0.92 \%$ as tip clearance changed from $10 \mathrm{~mm}$ to $20 \mathrm{~mm}$ and from $20 \mathrm{~mm}$ to $30 \mathrm{~mm}$, respectively. The maximum velocity decreased by $2.08 \%$ and $2.80 \%$ when tip clearance increased from $10 \mathrm{~mm}$ to $20 \mathrm{~mm}$ and from $20 \mathrm{~mm}$ to $30 \mathrm{~mm}$, respectively.

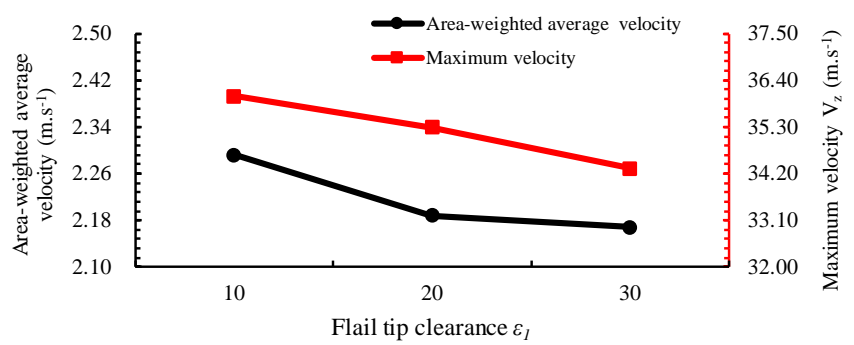

Fig. 28. Area-weighted average and maximum velocities at outlet under different flail tip clearances

The velocities at different sampling points under different flail tip clearances was shown in Fig. 29. The velocity increased from top to bottom of the outlet plane at the same tip clearance, and the velocity was generally symmetric of the center line. The velocity distribution curve was similar under different tip clearances at the top, middle and bottom of the outlet plane. The velocity at the top of the outlet plane was similar at the same tip clearance, and the velocity difference was small between different flail tip clearances. The velocity at middle of the outlet plane except at points 8 and 14 was similar at the same tip clearance, and the velocity at right and left extremities of the hood was larger than at other points. However, there was an obvious velocity variation at bottom of the outlet plane under the same flail tip clearance. This was similar to the results of decreased tip clearance would increase the velocity at outlet, found by Hu et al. [28] in a study of cross-flow fan.

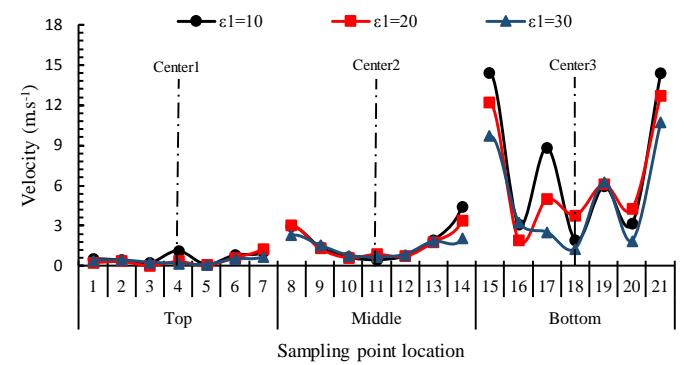

Fig. 29. Velocity at different sampling points under different flail tip clearances 
According to our observations, it can be concluded that an increase in flail tip clearance might degrade straw spreading performance.

\subsubsection{The E $\square$ ect of Flail Tip Clearance on Straw Flow-ability}

The streamlines on P1, P2 and P3 under different flail tip clearances were shown in Fig. 30. Vortices were distributed at the front section of the inlet region and the lower back of the hood with flail tip clearance of $10 \mathrm{~mm}$ (Fig. 30a). Both vortices dissipated when flail tip clearance was increased from $10 \mathrm{~mm}$ to $20 \mathrm{~mm}$. However, the streamline distribution changed a little as tip clearance was increased from $20 \mathrm{~mm}$ to $30 \mathrm{~mm}$. It can be concluded that an increase in flail tip clearance might improve straw flow-ability at the right and left extremities of the hood.

Fig.30b showed vortices distribution at the lower front of the hood when flail tip clearance was set at $10 \mathrm{~mm}$. The vortex at the lower front of the hood dissipated as the flail tip clearance was increased from $10 \mathrm{~mm}$ to $20 \mathrm{~mm}$, but a vortex materialized at the outlet. The size of the vortex region at the outlet decreased when tip clearance was increased from $20 \mathrm{~mm}$ to $30 \mathrm{~mm}$, but two other vortices appeared at the front of the hood. The results showed that an increase in flail tip clearance might reduce straw flow-ability in the middle area of the hood.

In Fig.30c vortices were distributed at the lower front and back of the hood. The vortex at lower front of the hood dissipated when flail tip clearance increased from $10 \mathrm{~mm}$ to $20 \mathrm{~mm}$. When the tip clearance changed from $20 \mathrm{~mm}$ to $30 \mathrm{~mm}$, the size of the vortex region at lower back of the hood decreased. The results showed that an increase in flail tip clearance might improve straw flow-ability around flail areas.
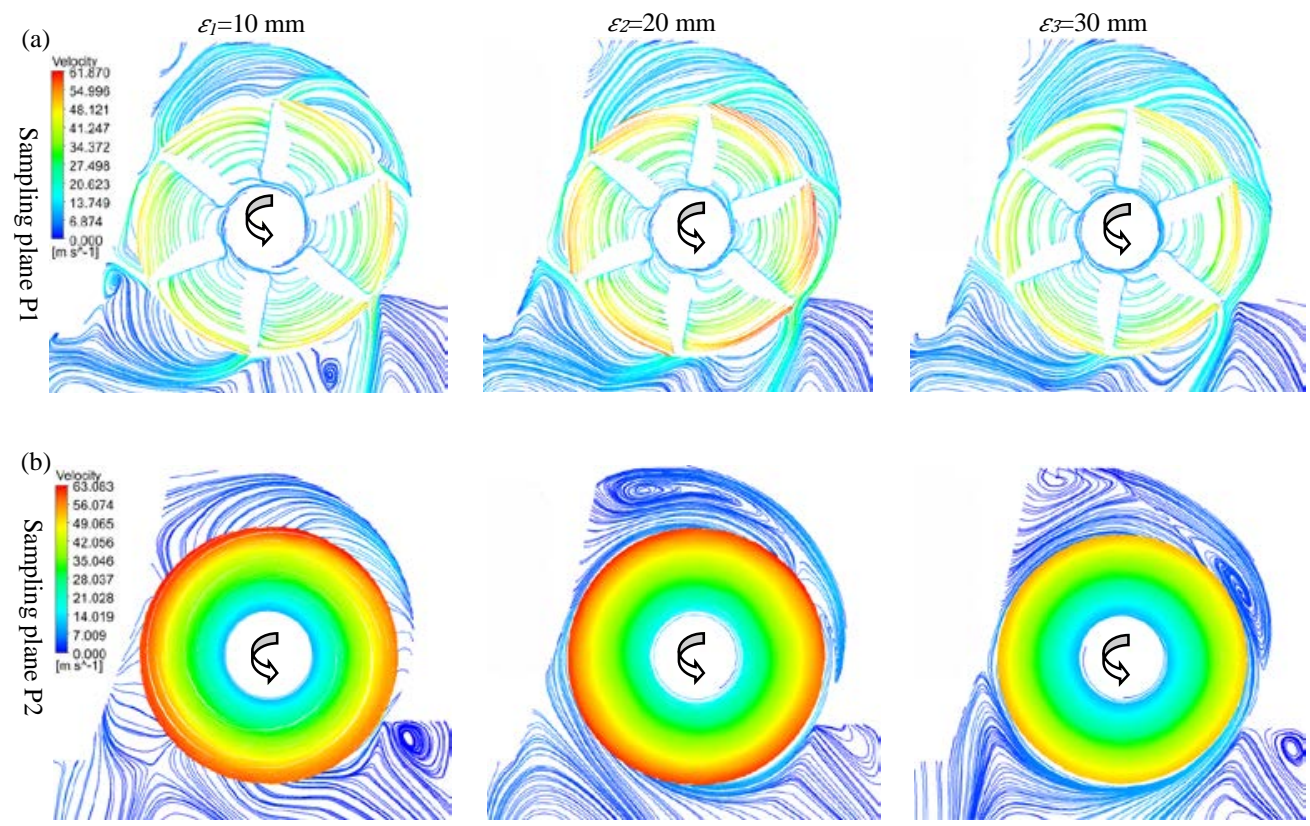

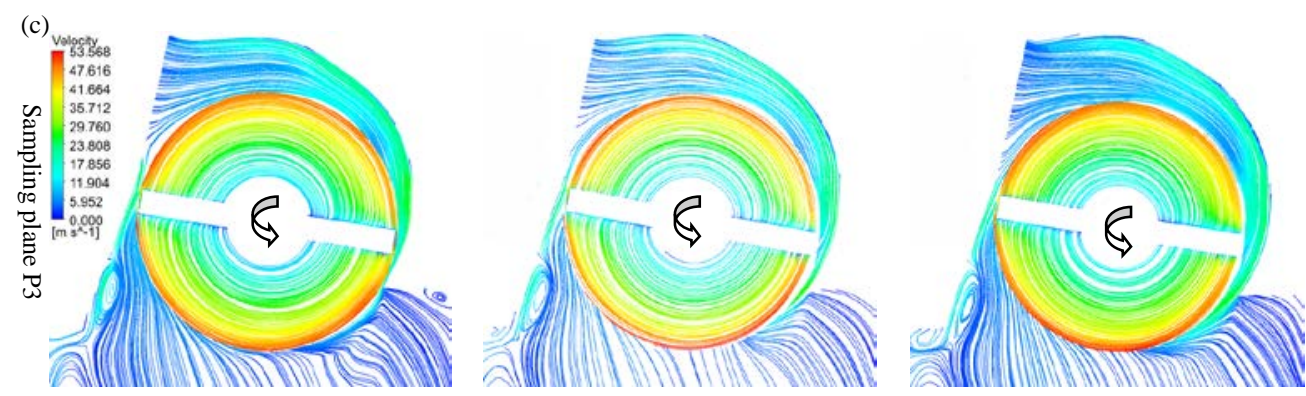

Fig. 30. Streamlines on P1, P2 and P3 under different flail tip clearances

According to the analysis of the three sampling sections, it can be concluded that an increase in flail tip clearance might improve overall straw flow-ability under the hood.

\section{Conclusions}

The effects of rotor speed, forward speed, hood clearance, fan blade number and flail tip clearance on flow field characteristics and performances of the tractor powered flail chopper were investigated via CFD numerical simulation. The following conclusions were drawn:

(1) Under all simulated conditions, large areas of negative pressure were produced at inlet of the flail chopper, velocities increased from top to bottom of the outlet plane, and the velocity was generally symmetric of center line at the outlet plane. With fan blades, the pressure at left and right extremities of the outlet plane was lower than surrounding areas under all simulatied conditions.

(2) The rotor speed and fan blade number were the main parameters to a tractor powered flail chopper performance. Under all simulated conditions, the greatest velocity at outlet is 38.96 $\mathrm{m} . \mathrm{s}^{-1}$.The increase in rotor speed and fan blade number improved straw feeding and spreading performance. Increased rotor speed could improve straw flow-ability under the hood, while an increase in fan blade number could reduce straw flow-ability.

(3) Under all simulated conditions, the greatest inlet flow rate was $0.6199 \mathrm{~kg} \cdot \mathrm{s}^{-1}$ when the flail tip clearance was $30 \mathrm{~mm}$. An increase in flail tip clearance appeared to improve straw feeding performance and straw flow-ability under the hood. The increase of rotor speed appeared to degrade the straw spreading performance.

(4) An increase in forward speed would degrade straw feeding performance and decrease straw flow-ability in the hood. The forward speed appeared to have little effect on the straw spreading performance.

(5) Under all simulated conditions, the greatest negative pressure at inlet is $35.53 \mathrm{~Pa}$ when the hood clearance was $110 \mathrm{~mm}$, and the greatest area-weighted average velocity at outlet was 2.97 $\mathrm{m} . \mathrm{s}^{-1}$ when the shell terrain clearance was $150 \mathrm{~mm}$. Too large or too small hood clearance will degrade straw feeding performance of flail choppers. The increase of hood clearance might improve the straw spreading performance of flail chopper and straw flow-ability.

\section{Acknowledgments}

This work was supported by the Special Fund for Agro-scientific Research in the Public Interest from the Ministry of Agriculture, China (Grant No. 201503136). 


\section{References}

1. Ding, H.L., Ma, X.Y., Li, J.R., et al.: Effects of cornstalk on organic matter content and microbial community structure in soil. Guizhou Agricultural Sciences. 44(10), 77-83 (2016) (in Chinese)

2. Prasad, R., Gangaiah, B., Aipe, K., Effect of crop residue management in a rice-wheat cropping systems on growth and yield of crops and on soil fertility. Experimental Agriculture. 35(4), 427-435 (1999)

3. Balwinder-Singh, E., Humphreys, P., Eberbach, et al.: Growth, yield and water productivity of zero till wheat as affected by rice straw mulch and irrigation schedule. Field Crops Research. 121, 209-225 (2011)

4. Qiu, J., Wu, M.L., Guan, C.Y., et al.: Design and experiment of chopping device with dynamic fixed knife coaxial for rice straw. Transactions of the CSAE. 31(10), 11-19 (2015) (in Chinese)

5. Singh, A., Dhaliwa, I., Dixit, A.: Performance evaluation of tractor mounted straw chopper cum spreader for paddy straw management. Indian Journal of Agricultural Research. 45(1), 21-29 (2011)

6. Chen, S.T., Zhou, X.J., Shi, Q.X., et al.: Experiment and Optimization on Factors Affecting the Performance of Wheat Stalk Macerator Smashing Devices. Transactions of the Chinese Society for Agricultural Machinery. 38(9), 67-70 \& 62 (2007) (in Chinese)

7. Shi, J.X., Chen, F., Guo, J. X., et al.: Design and experimental research of the field straw chopper with throwing cotton-stalk. Transactions of the CSAE. 22(3), 68-72 (2006) (in Chinese)

8. Jahr, A., Corves, B., Batos, A., et al.: Simulation of agricultural cutting and transport processes. Landtechnik. 65, 372-375 (2010)

9. Wu, D., Li, Y.K., Liu, H.S., et al.: Simulation of the flow characteristics of a drip irrigation emitter with large eddy methods. Mathematical and Computer Modelling. 58, 497-506 (2013)

10. Duga, T.A., Delele, A.M., Ruysen, K., et al.: Development and validation of a 3D CFD model of drift and its application to air-assisted orchard sprayers. Biosystems engineering. 154, 62-75 (2017)

11. Qin, W.C., Xue, X.Y., Cui, L.F., et al.: Optimization and test for spraying parameters of cotton defoliant sprayer. International journal of agricultural and biological engineering. 9(4), 63-72 (2016)

12. Ayli, E., Celebioglu, K., Aradag, S.: Determination and generalization of the effects of design parameters on Francis turbine runner performance. Engineering Applications of Computational Fluid Mechanics. 10 (1), 547-566 (2016)

13. Zhang, C., Li, G.Y.: Numerical Simulation of Regulating Performance of Direct-Operated Pressure Regulator for a Microirrigation Lateral. CCTA 2014, IFIP AICT. 452, 285-303 (2015) 
14. Shen, P.Y., Zhao, H., Zhang, Y.Z.: Numerical Simulation of Fluid Field for High-speed Cutting Grinding. Transactions of the Chinese Society for Agricultural Machinery. 41 (9), 60-65 (2010) (in Chinese)

15. Wang, X.N., Li, C.Q., Shi, J.X., et al., Research on Simulating the Wind Field of the Field Straw Chopper with Throwing Cotton Stalk. Transactions of the Chinese Society for Agricultural Machinery. 38 (8), 67-69 (2007) (in Chinese)

16. Chon, W., Amano, R.S.: Investigation of Flow Behavior around Corotating Blades in a Double-Spindle Lawn Mower Deck. International Journal of Rotating Machinery. 1, 77-89 (2005)

17. Jia, H.L., Wang, L.C., Li, C.S., et al.: Combined stalk-stubble breaking and mulching machine. Soil Tillage Research. 107, 42-48 (2010)

18. Mao, H.P., Chen, C.Y.: Working Mechanism and Parameters Determination of Field Straw Chopper. Transactions of the CSAE. 11(4), 62-66 (1995) (in Chinese)

19. Sahili, A., Zogheib, B., Barron, M.R.: 3-D Modeling of Axial Fans. Applied Mathematics. 4632-651 (2013)

20. Brown, M.: Velocity Measurements Near an Automotive Cooling Fan. MASc Thesis, University of Windsor, Windsor, 2001

21. Rohdin, P., Moshfegh, B.: Numerical predictions of indoor climate in large industrial premises. A comparison between different k- $\varepsilon$ models supported by field measurements. Building and Environment. 42, 3872-3882 (2007)

22. Dogruoz, M.B., Shankaran, G.: Computations with the multiple reference frame technique: Flow and temperature fields downstream of an axial fan. Numerical Heat Transfer. 71 (5), 488-510 (2017)

23. Zheng, Z.Q., He, J., Li, H.W., et al.: Design and Experiment of Straw-chopping Device with Chopping and Fixed Knife Supported Slide Cutting. Transactions of the Chinese Society for Agricultural Machinery. 47 (S1), 108-116 (2016) (in Chinese)

24. Ma, Y.H., Zhang, J.X., Cheng, F.: Performance Experiments of Field Straw Chopper for Mulching or Reclaiming. Journal of Agricultural Mechanization Research. 4, 126-128 (2009) (in Chinese)

25. Zhang, J.X., Wang, X.L., Chen, F., et al.: Study on Working Parameters of Knife Roller of Field Straw Chopper for Mulching or Reclaiming. Transactions of the Chinese Society for Agricultural Machinery. 38 (6), 82-85\&37 (2007) (in Chinese)

26. Zhang, Z. Q., McHugh, A. D., Li, H. W., et al.: Global overview of research and development of crop residue management machinery. Applied Engineering in Agriculture. 33 (3), 329-344 (2017)

27. Yu, J.Z., Yu, Y.C., Zhu, X.X., et al.: Parameter determination and examination of the 4Q-1.5-type straw stalk grinder main working part. Journal of Agricultural Mechanization Research. 9, 74-76 \& 79 (2006) 
28. Hu, J.W., Ding, G.L., Zhao, L., et al.: Simulation of flow field and performance of cross-flow fan. Chinese journal of mechanical engineering. 40 (4), 62-66 (2004) (in Chinese) 